\title{
Creative Figure Design in Technical and Vocational Education
}

\author{
Rui-Lin Lin \\ Department of Commercial Design \\ Chienkuo Technology University \\ No. 1, Chieh Shou N. Rd., Changhua City 500, Taiwan \\ email: linrl2002@gmail.com
}

\begin{abstract}
With the students from two first-grade classes who entered the Technology University in Central Taiwan in 2011 as the research subjects, this study integrated the operator's requirements for innovative figures for socks into the teaching and learning processes of the creative design curriculums. The teacher inspired the students' creative ideas through creative thinking and methods and adopted the individual discussion teaching method to discuss the students' works with them and help them to make modifications. The operator could reference the design results to develop new products with creative figures.
\end{abstract}

Keywords- innovative product, figure design, creative design

\section{INTRODUCTION}

The focus of design education is on the educational goal of combining theories and practices. And improving students' abilities of oral presentation and writing copy writers is essential. When teachers teach courses related to creative thinking design and idea development, they all hope to integrate design issues from industries to teaching and learning processes of these professional design courses, for the purposes of training students' practical abilities and helping them to get familiar with how things work in job fields to make preparations for their future careers.

Secondly, if teachers would like to integrate design issues from industries into teaching and learning of their courses, they have to make earlier preparations or preserve a few weeks as flexible time, for mechanisms of cooperation between academia and industry to work. Of course, vendors have to explain to teachers and students their design requirements, limitations on product manufacturing, and related regulations, so that teachers and students can develop creative ideas for innovative products within proper ranges.

The vendor which commissioned this study to design was founded in 1979. In 1983, it started to develop its own brand. However, its main focus has still been manufacturing services. With regard to the continuous improving social and living standards and the trend of consumers pursuing product quality and novelty, the vendor has continuously developed new products and spent efforts in material R\&D and making innovative improvements in design. From the manufacturing industry to the service industry, the vendor finally upgraded its old factory to a tourism sock factory, in hopes of, through demonstration of innovative sock products, tourists learning more about socks and getting to know the local industry, so that the spirit and services of innovative entrepreneurship and sustainable management can be implemented in the traditional industry via tourism [7].

\section{LITERATURE REVIEW}

With the advancement of technology, innovative products have been developed in a very fast pace, as a result, shortening life cycles of new products. Development of new product technology and innovation of designs must meet consumers' demands with consideration of experts' opinions and analyses, so that more guarantee can be brought to R\&D of new products [3]. In processes of innovative product design and manufacturing, enterprises must always pay attention to consumers' psychological changes and demands which are always varying. And they must follow standards of innovative technology and business development. Thus, engineering and designing departments are getting more and more severe challenges. In this kind of environment, enterprises must continuously innovate in the aspect of their products and study related knowledge and principles, to help their departments develop new technology and innovative new products [6].

Some scholar applied the TRIZ innovative thinking method to develop products to help designers break limitations on product manufacturing and bottlenecks, combined theories and practices along with case selection and operation, created new principles and methods, and improve manufacturers' manufacturing quality [1]. There was also an application with the industrial module of manufacturing computer aided products to propose application models based on different user demands. This way, model building demands could be met. And analyses for product craft design and computer aided model building frames were conducted. The research result was successful. The multi-dimensional models could be connected with each other for analyses. And the parameters were regressed on the model building frame to come up with a solution to help the system achieve the highest efficiency in development [5].

\section{InNOVATIVE PRoduct Design}

Creative thinking teaching activity design is based on the Theory of Creativity, and teachers can set up learning goals according to cognition of creativity development and affective aspects. In this study, the innovative product design issue was to come up with ideas of cute and rakish innovative figure designs, through the innovative thinking of the teacher and the students, as references for the vendor to develop its products. 


\section{A. Meanings and Characteristics}

Design is an idea of completing a specific action through thinking. Creative thinking teaching activity design is a teaching activity idea to develop students' creative thinking abilities. Teachers should apply creative thinking strategies or principles, through a series of creative activity themes, to inspire students' creativity based on plans. Therefore, teachers must take teaching themes, contents of teaching materials, students' levels, teaching strategies, teaching evaluation, etc. into consideration.

The characteristics include: (1) having a teaching goal of developing students' creativity; (2) applying creative thinking techniques or principles as a strategy; (3) flexible, diversified, and active applications considering interest; and (5) diversified and adaptive assessments not limited to written format [4].

\section{B. Creative Thinking and Teaching}

Creation includes innovative, novel, and unique thinking or a new invention. Creation is to come up with a new idea, a new solution, a new program, a new product, or a new system when facing a difficulty. Creation is a type of ability, as well as a process. The features of creativity include: (1) smoothness of thoughts; (2) flexibility of adjusting to changing circumstance; (3) uniqueness of proposing unique solutions for problems; and (4) preciseness of insightful analyses and careful consideration.

Creation is a thinking process, with stages including (1) preparative stage, (2) incubational stage, (3) illumination stage, and (4) verification stage. The factors which may influence creativity are (1) intelligence (people with low intelligence tend to have less creativity), (2) thinking method, (3) personality, (4) traditional social culture, and (5) education method. And the teaching strategies teachers can apply are: (1) brain storming, (2) encouraging association, (3) making partial changes, (4) checklist method, (5) combination method, (6) attribute listing, (7) encouraging students' divergent thinking, (8) allowing students to express different opinions, and (9) building students' creative characters [2].

\section{DESIGN RESUlts}

The teacher and the students designed the creative figures based on the requirements. These works were scored by the teacher and the vendor based on (1) design (35\%), (2) creativity (35\%), and color (30\%), and the ones with higher scores were selected as references for the vendor to development its products.
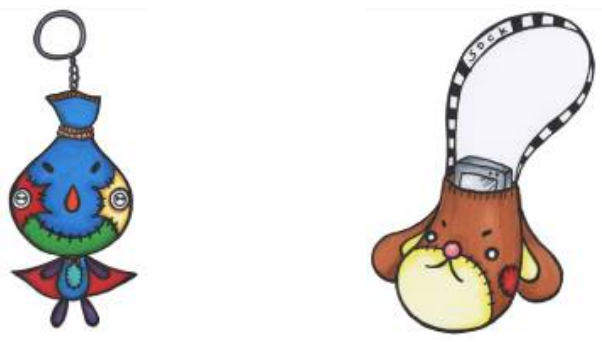

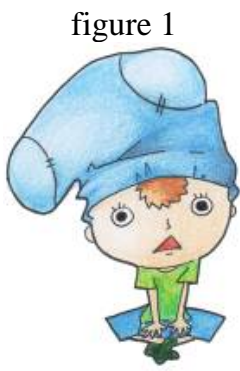

figure 3

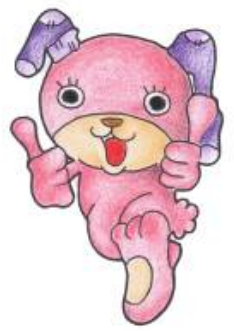

figure 5

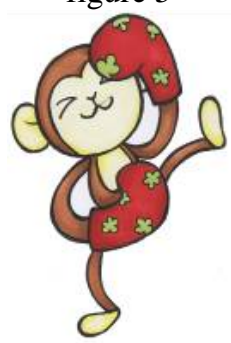

figure 7

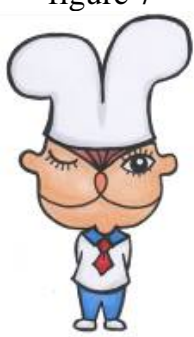

figure 9

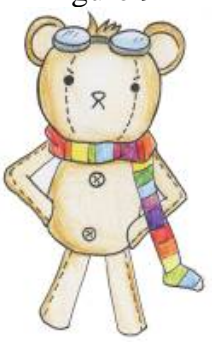

figure 11

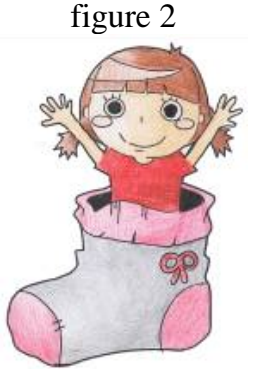

figure 4

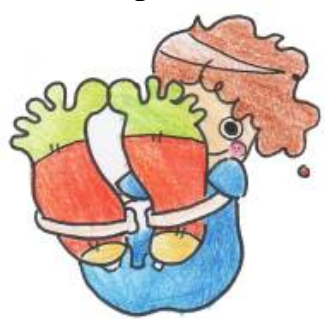

figure 6

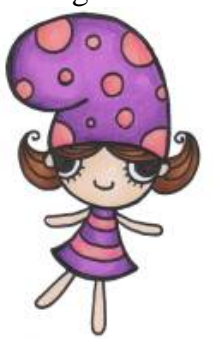

figure 8
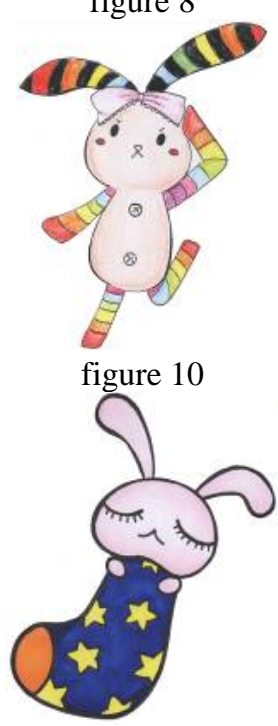

figure 12 


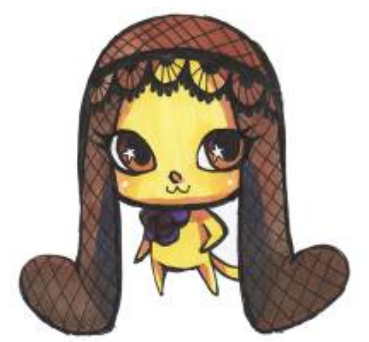

figure 13

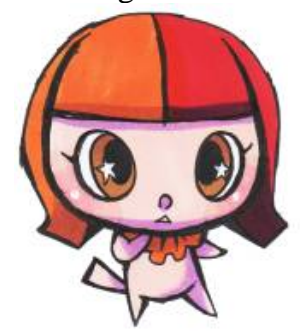

figure 15
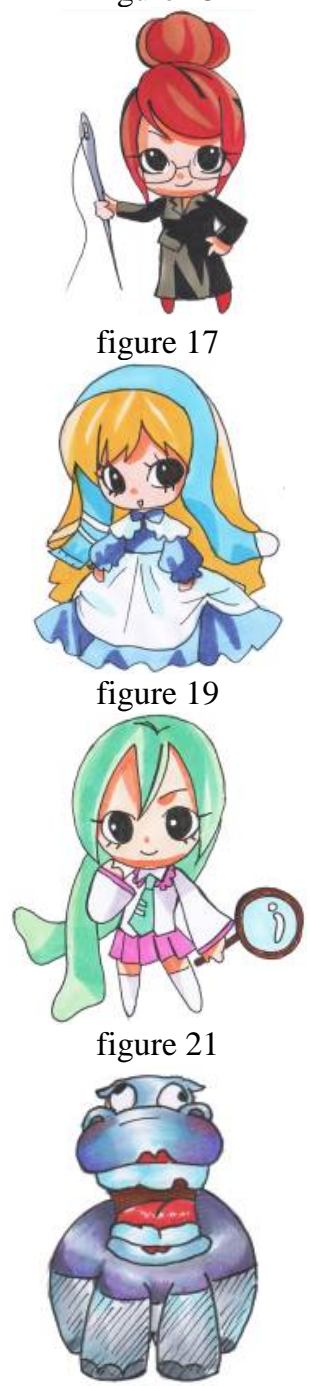

figure 23

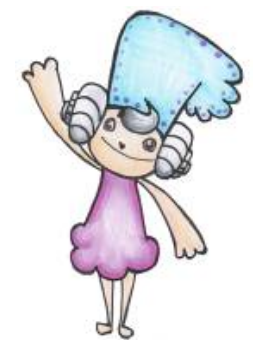

figure 14

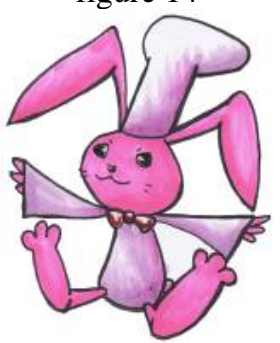

figure 16

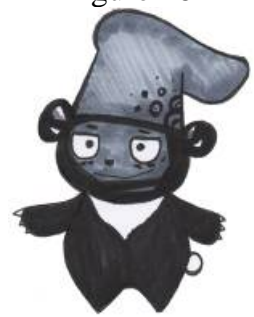

figure 18

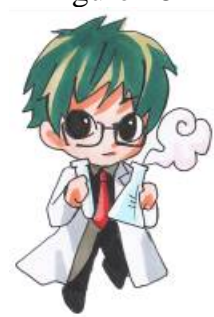

figure 20
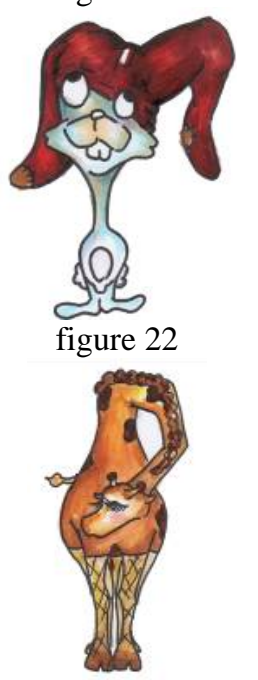

figure 24

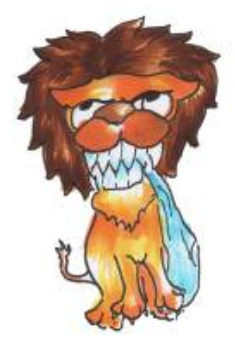

figure 25

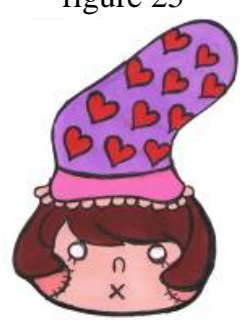

figure 27
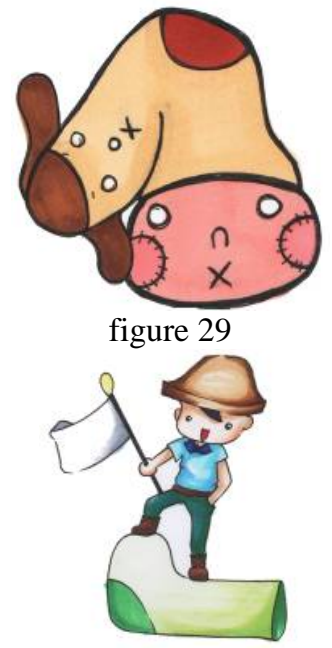

figure 31

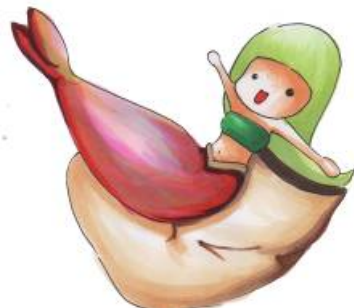

figure 33

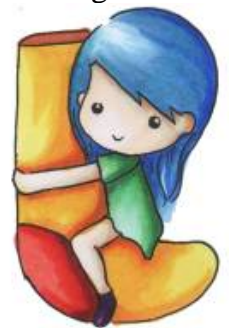

figure 35

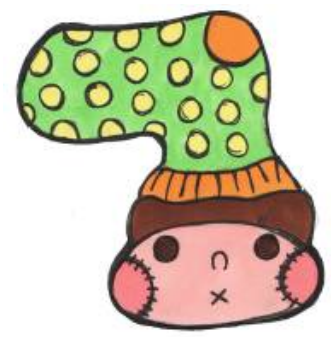

figure 26
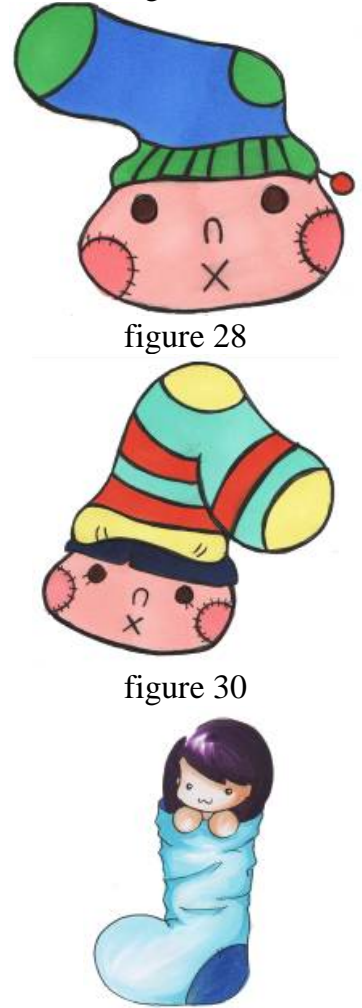

figure 32

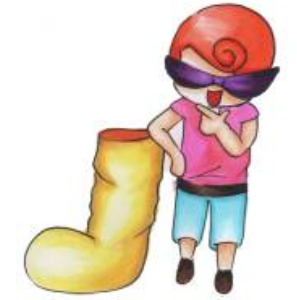

figure 34

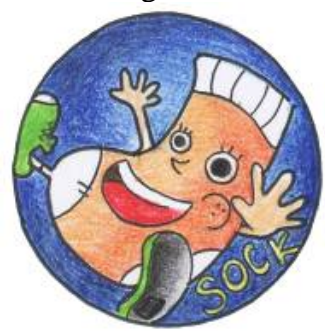

figure 36 


\section{CONCLUSIONS}

In this study, according to the vendor's requirements regarding innovative product design, several figures were created as references for the vendor's product $R \& D$. Generally speaking, this cooperation was helpful for the teacher, the students, and the vendor. It is worthy to be continuously promoted in the future.

\section{A. Discussions}

(1) Students: The students created the innovative figures for product designs under the teacher's guidance based on the information provided by the vendor regarding the manufacturing procedure of products and its design requirements. Through this experience, their practical design abilities were practiced and they were able to get to know how things actually worked in the industry, which was helpful for their future career preparation.

(2) Teacher: The teacher was commissioned by the vendor to integrate its issues into the creative design course, achieving the teaching goal of combining theories and practices. And this was a chance to make contact with the industry, which was helpful to improve the teacher' s professional competence.

(3) School: The cooperation between the academia and the industry was helpful for the students to develop their abilities for future careers and for the teacher to improve practical teaching abilities. Generally speaking, this cooperation was of great benefits to the school.

(4) Vendor: The vendor accepted the ideas for innovative product design provided by the teacher and the students from the school. Although it was not easy to manufacture these products immediately, these ideas could still be used as references for designs of product styles. The vendor was actually the biggest winner in this cooperation.

B. Suggestions

(1) The students could improve their practical abilities through this kind of cooperation between the academia and the industry and develop the attitude of not being afraid of tough challenges, for the purpose of making early preparation for future careers.

(2) The teacher could make more contacts with vendors in industries to learn more about different requirements and issues encountered in various industries, also to improve personal professional abilities in field works.

(3) The school should encourage teachers to lead their students to participate in academia-industry cooperation projects more, in order to obtain more external resources to create an environment of product innovative design to improve the school' $\mathrm{s}$ image. Of course, the school should offer more rewards so that teachers and students would be more willing to participate in academia-industry cooperation projects.
(4) The vendor obtained the creative ideas and designs from the teacher and the students from the school. Although there are still some follow-up manufacturing issues to be solved for these creative ideas, those wonderful and creative image designs made by the teacher and the students were still very helpful. The vendor should offer the teacher and the students some necessary feedbacks or rewards timely.

\section{REFERENCES}

[1] A. Albers, M. Ohmer, T. Alink, Accessibility of the Innovative Principles to Further Levels of Abstraction in Product Development, Procedia Engineering, vol. 9, 2011, pp. 222-235.

[2] C. X. Ye, Educational Psychology, Psychological Publishing Co., Ltd., Taipei, 2011.

[3] F. M. Tseng, Y. T. Lin, S. C. Yang, Combining Conjoint Analysis, Scenario Analysis, the Delphi Method, and the Innovation Diffusion Model to Analyze the Development of Innovative Products in Taiwan's TV Market, Technological Forecasting and Social Change, vol 79, Issue 8, October, 2012, pp. 1462-1473.

[4] L. A. Chen, Theories and Practices of Creative Thinking Teaching, Psychological Publishing Co., Ltd., Taipei, 2008.

[5] M. Heitzig, G. Sin, P. Glarborg, R. Gani, A Computer-aided Framework for Regression and Multi-scale Modelling needs in Innovative Product-process Engineering, Computer Aided Chemical Engineering, vol 28, 2010, pp. 379-384.

[6] N. Janthong, D. Brissaud, S. Butdee, Combining Axiomatic Design and Case-based Reasoning in an Innovative Design Methodology of Mechatronics Products, CIRP Journal of Manufacturing Science and Technology, vol. 2, Issue 4, 2010, pp. 226-239.

[7] The Soxlink Functional Wear Tourism Factory, http://www.soxlink.com.tw/soxking/, 2013, 3/22. 